\title{
Image Registration and Fusion for Interventional MRI Guided Thermal Ablation of the Prostate Cancer
}

\author{
Baowei Fei ${ }^{1}$, Zhenghong Lee ${ }^{1,2}$, Daniel T. Boll ${ }^{2}$, \\ Jeffery L. Duerk ${ }^{1,2}$, Jonathan S. Lewin ${ }^{1,2,3}$, and David L. Wilson ${ }^{1,2}$ \\ ${ }^{1}$ Department of Biomedical Engineering \\ Case Western Reserve University, Cleveland, OH 44106, USA \\ $\{$ BXF18, DLW\} apo. cwru.edu \\ ${ }^{2}$ Department of Radiology \\ University Hospitals of Cleveland, Cleveland, OH 44106, USA \\ \{Lee, Boll, Duerk, Lewin\}@uhrad.com \\ ${ }^{3}$ Departments of Oncology and Neurological Surgery, \\ University Hospitals of Cleveland, $\mathrm{OH} 44106$, USA
}

\begin{abstract}
We are investigating interventional MRI (iMRI) guided thermal ablation treatment of the prostate cancer. Functional images such as SPECT can detect and localize tumor in the prostate not reliably seen in MRI. We intend to combine the advantages of SPECT with iMRI-guided treatments. Our concept is to first register the low-resolution SPECT with a high resolution MRI volume. Then by registering the high-resolution MR image with iMRI acquisitions, we can, in turn, map the functional data and high-resolution anatomic information to iMRI images for improved tumor targeting. For the first step, we used a mutual information registration method. For the latter, we developed a robust slice to volume (SV) registration algorithm. Image data were acquired from patients and volunteers. Compared to our volume-tovolume registration that was previously evaluated to be quite accurate, the SV registration accuracy is about $0.5 \mathrm{~mm}$ for transverse images covering the prostate. With our image registration and fusion software, simulation experiments show that it is feasible to incorporate SPECT and high resolution MRI into the iMRI-guided treatment.
\end{abstract}

\section{Introduction}

We use an interventional magnetic resonance imaging (iMRI) system to guide minimally invasive treatments, including the radiofrequency (RF) thermal ablation of abdominal cancers. [1-3] The iMRI system consists of a $0.2 \mathrm{~T}$, clinical C-arm open MRI scanner, an in-room RF-shielded liquid crystal monitor, an MR compatible mouse, a foot pedal, and a RF device. We are currently investigating the extension of these techniques to the treatment of prostate cancer. Since MRI does not reliably show prostate tumors, we intend to incorporate nuclear medicine images with higher sensitivity for detecting and localizing prostate tumors. [4,5] We will first register the low-resolution functional SPECT images with a high resolution MRI volume. Then by registering the high-resolution MR volume with iMRI acquisitions, we can, in turn, map the functional data and high-resolution anatomic information to iMRI images for 
improved tumor targeting. If this procedure is successful, then a variety of potential visualization tools can help the physician appropriately localize and apply treatments.

In next sections, we will report a three dimensional registration method for SPECT and high resolution MRI volumes, a slice to volume registration algorithm for iMRI thick slices and high resolution MRI volume, and image registration and fusion software for potential applications in iMRI-guided thermal ablation of the prostate.

\section{Registration Algorithms}

\subsection{Registration of SPECT and High Resolution MRI Volumes}

A mutual information algorithm was applied in this study to the registration of pelvic image volumes from MRI and SPECT for potential use in prostate cancer diagnosis, staging, and treatment planning. Mutual information (MI) was chosen because of its potential to align multi-modality images [6,7]. Registration of SPECT and MR images is challenging because the two image types have very different spatial resolutions and image features. The pelvic region is difficult for alignment between images from the two scans. Before registration, both SPECT and MRI volumes were resized using trilinear interpolation to create volumes matrix of $128 \times 128 \times 128$ with $3 \mathrm{~mm}$ isotropic voxels, a voxel size between that of the two scans. The standard parameter set for automatic registration include: 256 intensity levels for each volume, the entire 2D joint histogram, the full field of view of 128x128x128 voxels for both volumes, and no masking or cropping of either volume.

\subsection{Registration of iMRI Slice and High Resolution MRI Volume}

We used two similarity measures, mutual information [8] and correlation coefficient (CC), [9] in our registration. We used a similar algorithm as previously reported by us. [10] We use a multi-resolution approach and perform registration from low to high resolution. We use correlation coefficient at the two lower resolutions because it gives fewer local maximums and because it can be calculated faster than MI. [11] We use MI at full resolution because the peaked similarity function gives a more precise solution than CC. [12] To avoid local maximums, we include a restarting feature where registration is restarted with randomly perturbed parameters obtained from a uniform distribution about the initial transformation values at the current resolution being used. [13] The algorithm restarts until the absolute $\mathrm{CC}$ is above an experimentally determined threshold or the maximum number of restarts is reached.

\section{Experimental Methods}

\subsection{SPECT Image Acquisition}

We acquired SPECT images from three patients S1-S3 with either high Gleason scores ( $>5$ ) from biopsy or rising PSA level ( $>10 \mathrm{mcg} / \mathrm{L}$, prostate specific antigen) or palpatation staging beyond stage T1. After patient eligibility was established, patients gave informed consent. The Institutional Review Board of the University Hospitals of Cleveland approved the imaging protocol. The radiotracer used for SPECT imaging is 
ProstaScint ${ }^{\circledR}$ (Cytogen Corporation, Princeton, NJ), a monoclonal antibody that binds to prostate-specific membrane antigen (PSMA).

Approximately four days after injecting $5 \mathrm{mCi}$ ProstaScint ${ }^{\circledR}$, the abdominal region and pelvic region were scanned using a two-head Siemens E.CAM ${ }^{+}$camera (Siemens Medical System, Inc., Hoffman Estates, IL). The evening before the scanning, patients were asked to perform a bowel prep with Fleet R Prep Kit \#3 (Fleet Pharmaceuticals, Lynchburg, VA). Images were acquired with a medium energy collimator and $15 \%$ energy window. The acquisition parameters included a step-andshoot motion, a $128 \times 128$ pixel matrix for each projection, an imaging time of $25 \mathrm{sec}$ per stop, and a total of 120 stops over a full $360^{\circ}$ rotation. The field of view of was 53.3 x 38.7-cm. The iterative image reconstruction algorithm OSEM (ordered subsets expectation maximization) [14] was used to reconstruct the SPECT images with 8 subsets and 10 iterations. The SPECT images were comprised of isotropic voxels with size of $4.8 \times 4.8 \times 4.8-\mathrm{mm}$. Each patient had one SPECT scan of the pelvis.

\subsection{High Resolution MR Image Acquisitions}

High-resolution MRI volumes were acquired using a $1.5 \mathrm{~T}$ Siemens MRI system (Magnetom Symphony, Siemens Medical Systems, Erlangen, Germany). An 8element phased array body coil was used to ensure coverage of the prostate with a uniform sensitivity. Typically two anterior and two posterior elements were enabled for signal acquisition. We used two different MR sequences.

First, we used a 3D rapid gradient echo sequence (PSIF) designed to acquire the spin-echo component of the steady state response, rather than the free induction decay. The spin echo component forms immediately prior to the RF pulse; it is shifted toward the prior RF pulse through appropriate gradient waveform design. The sequence with 9.4/5.0/60 (TR/TE/flip) yields 160x256x128 voxels over a 219x350x192-mm rectangular FOV. There is over sampling at $31 \%$ in the slice direction to reduce aliasing artifacts. The acquisition time is $4 \mathrm{~min}$ and $15 \mathrm{sec}$. This sequence gave excellent image contrast for the prostate and its surroundings. It was used to acquire volumes for volunteers S4-S6. Second, we used a 3D RF spoiled gradient echo steady state pulse sequence (FLASH) with TR/TE/flip parameters of 12/5.0/60 which give 256 × 256 x 128 voxels over a 330x330x256-mm field of view (FOV) to yield $1.3 \times 1.3 \times 2.0-\mathrm{mm}$ voxels oriented to give the highest resolution for transverse slices. The acquisition time is $5 \mathrm{~min}$ and $38 \mathrm{sec}$. This sequence is good for pelvic imaging but is not ideal for the prostate. It was used to acquire volumes for patients S1-S3.

When acquiring MR volumes, volunteers laid supine in a manner similar to the diagnostic position in routine MR scanning. Between volume acquisitions, volunteers got up from the MR table, stretched, and walked around to ensure that they would assume a different position when they laid back on the table. The coil array was centered on the prostate. We acquire one volume from each of patients S1-S3 and three volumes from each of volunteers S4-S6. 


\subsection{Interventional MRI Image Acquisitions and Simulation}

We acquired iMRI images using a clinical 0.2 T C-arm open MR scanner (Siemens Open Symphony, Erlangen, Germany) modified for interventional MRI procedures and in this paper referred to as the iMRI system. We used a 3D PSIF with 25/13/60 (TR/TE/FA) for image volume acquisitions and two-dimensional (2D) PSIF with 15.2/7.4/45 (TR/TE/FA) for image slice acquisitions. The iMRI volumes were $256 \times 256 \times 100$ with voxel size of $1.3 \times 1.3 \times 1.4 \mathrm{~mm}$. The iMRI slices were $128 \times 128$ with in-plane pixel size of $2.8 \times 2.8 \mathrm{~mm}$ and with effective slice thickness of $5 \mathrm{~mm}$.

We acquired iMRI images under the conditions simulating the treatment application. The volunteer was supine, and his legs were supported at $30^{\circ}-60^{\circ}$ relative to the horizon and separated in a "V" with an angle of $60^{\circ}-90^{\circ}$ between two legs. This is similar to the lithotomy position used in prostate therapies, and it should provide access for needle insertion in brachytherapy or RF thermal ablation. We call this the treatment position. For each of the volunteers S4-S6, we acquired two volumes and 30 iMRI image slices covering the prostate. They included 10 transverse, 10 coronal, and 10 sagittal image slices. We call these images "actual" iMRI images to differentiate them from later experiments using "simulated" iMRI slices.

To test a variety of clinical conditions, we used high-resolution MRI volumes to simulate iMRI images by creating thick slices and adding noise and receive coil inhomogeneity. Clinically, we typically use an iMRI slice thickness of $4.0-6.0 \mathrm{~mm}$. We averaged 3 slices $1.4 \mathrm{~mm}$ thick to create a $4.2 \mathrm{~mm}$ thick slice. We added noise to the simulated iMRI image. [15,16]

\subsection{Registration and Fusion Experiments}

We performed three dimensional MI registration of MRI and SPECT images using three pairs of data sets from patients S1-S3. Before registration, we preprocessed both MR and SPECT images such as intensity scaling and isotropic processing.

We used nine pairs of high-resolution MR volumes to perform simulated slice to volume registration experiments. For each volume pair, we extracted data from one volume to simulate thick iMRI image slices; and then we registered the simulated image slices to the other volume. We desire an iMRI slice image acquisition method that gives robust, accurate registrations and is relatively insensitive to acquisition parameters. Hence, we performed experiments to determine the dependence on slice orientation (transverse, sagittal and coronal), on slice position relative to the prostate (above, centered, and below) and on image noise from fast imaging techniques.

We performed SV registration experiments using actual iMRI images. We visually evaluated results. For each volunteer S4-S6, there were three high-resolution MR volumes and 30 iMRI image slices giving $90 \mathrm{SV}$ registration experiments, and a total of 270 experiments.

We simulated the iMRI-guided procedures using our image registration and fusion software that are specially designed for this application. Before treatment, we acquired SPECT and high resolution MRI volumes from the same patient. Second, we registered the two images and transferred the pair of aligned data sets to a workstation that was used for slice to volume registration. Third, we connected the workstation to 
the iMRI scanner and obtained iMRI image slices from the scanner. Fourth, we performed slice to volume registration. Finally, the software created fused images of the three modalities for image guidance. All registration and fusion are automatic.

\subsection{Registration Evaluation}

Although we evaluated registration using a variety of quanitative measures, visual inspection was also employed. We used RegViz, a program created in IDL in our laboratory with multiple visualization and analysis methods. First, we manually segmented prostate boundaries in image slices and copied them to corresponding slices. This enabled visual determination of the overlap of prostate boundaries over the entire volume. Second, color overlay displays were used to evaluate overlap of structures. To visualize potential differences, it was quite useful to interactively change the contribution of each image using the transparency scale. Third, we used a sector display, which divided the reference and registered images into rectangular sectors and created an output image by alternating sectors from the two input images. Even subtle shifts of edges would be clearly seen.

Registration between SPECT and MRI was devaluated by comparing automatic registration results to manual registration, our gold standard. Manual registration was done by two board-certified nuclear medicine radiologists who were blinded to the automatic registration results prior to performing registration. Manual registration was done using a software package with a graphical user interface (GUI) developed inhouse, which allows graphical manipulation of volumes with six degrees of freedom in a rigid body registration. A successful automatic registration is defined as when the transformation parameters are almost equal to that of the manual registration. Displacements must be $<2$ voxels $(6 \mathrm{~mm})$ in the $\mathrm{x}, \mathrm{y}$, or $\mathrm{z}$ directions and $<2$ degree in rotation for the three angles measured about each of the 3 axes.

The standard evaluation method for the slice to volume registration was to compare $\mathrm{SV}$ and volume to volume (VV) registration. [17] It is quite reasonable to use VV registration as our gold standard because it was previously identified to be quite accurate. For volume pairs acquired over a short time span from a supine subject with legs flat on the table, following VV registration, prostates were well aligned and prostate centroid displacements typically $<1 \mathrm{~mm}$. The VV registration accuracy as determined from displacements of pelvic bony landmarks was $1.6 \pm 0.2 \mathrm{~mm}$. To compare SV and VV registration, we defined a rectangular volume of interest (VOI) just covering the prostate and calculated voxel displacements between the two registrations. We report the average voxel displacement as an error measure. In addition, we defined the SV registration as being successful when the 3D displacement was less than $2.0 \mathrm{~mm}$.

\section{Results}

\subsection{Registration of iMRI Slice to High Resolution MRI Volume}

We reported SV registration results for slices near the prostate in the three standard orthogonal orientations. Comparing to $\mathrm{VV}$, mean and standard deviation registration errors across 9 volume pairs and $60 \mathrm{SV}$ registration experiments were $0.4 \mathrm{~mm} \pm 0.2$ 
$\mathrm{mm}, 0.5 \mathrm{~mm} \pm 0.2 \mathrm{~mm}$, and $2.6 \mathrm{~mm} \pm 1.6 \mathrm{~mm}$ for transverse, coronal and sagittal slices covering the prostate, respectively. Transverse slices worked best because they contain many relatively rigid anatomical structures.

Registration experiments with simulated iMRI images showed the SV registration was very insensitive to noise. We performed over 100 registration experiments with noise added to give signal to noise ratio (SNR) ranging from 20 to 5 . Using the slice configurations recommended above (transverse slices near the prostate center), we obtained $100 \%$ successful registrations (an error $<2.0 \mathrm{~mm}$ ) for SNR's $\geq 10$, a value much worse than the clinical SNR value of $\approx 25$ on our iMRI system.

For registration of actual iMRI image slices and a high-resolution MR volume, the contours overlap and overlay images show that the prostate matches very well. Other visual inspection techniques also demonstrate excellent registration. Note that a single iMRI image was used to produce this registration result.

The time for an SV registration was typically about $5 \mathrm{sec}$ on a Pentium IV, $1.8 \mathrm{GHz}$ CPU, with 1Gbytes of memory. The algorithm was written in IDL (Interactive Data Language, Research System Inc., CO) and could probably be made much faster in a lower level language such as C.

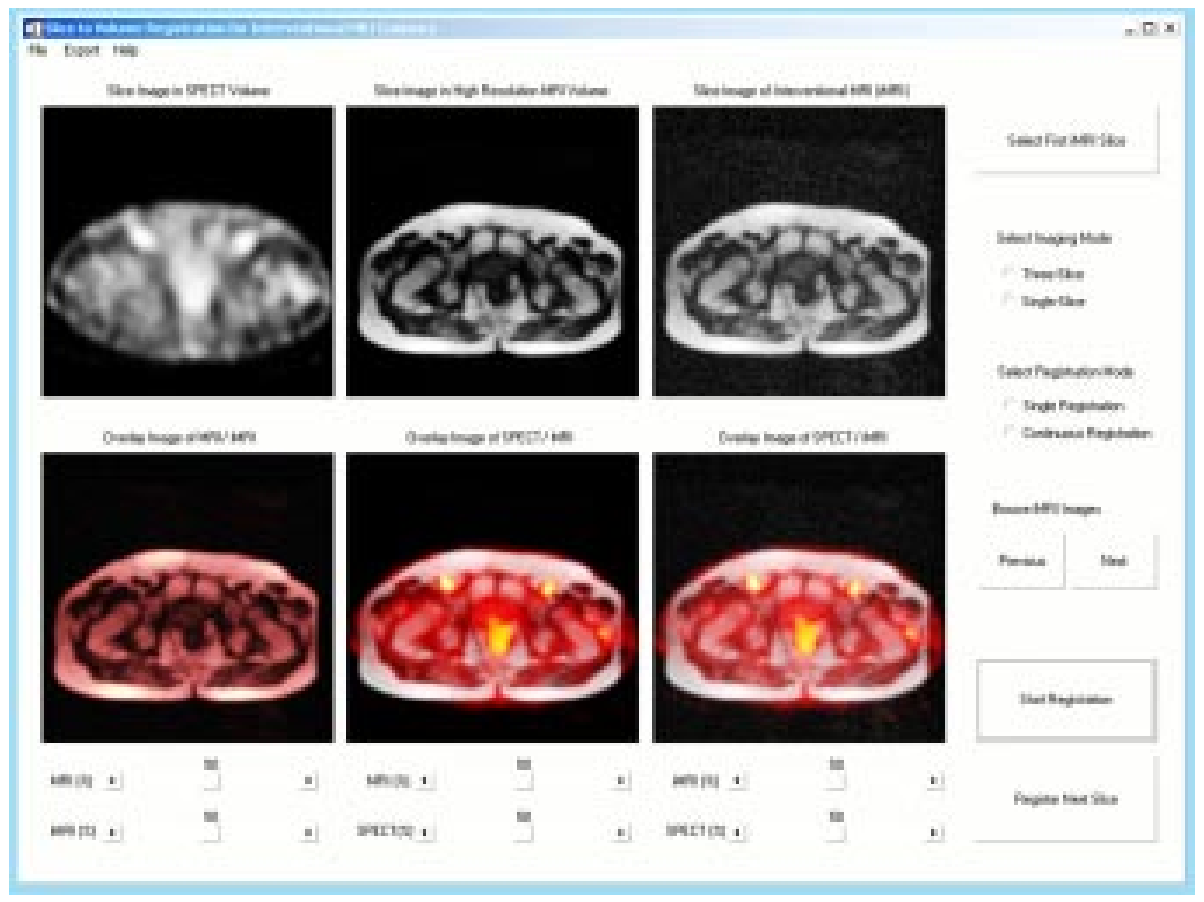

Fig. 1. User interface of the image registration and fusion software. The top three windows from left to right show corresponding registered SPECT, high resolution MRI, and iMRI images, respectively. The bottom three windows from left to right show the fused images, iMRI/MRI, SPECT/MRI, and SPECT/iMRI, respectively. Other buttons and sliders control the configuration and registration. High resolution MRI and SPECT images are from the patient S1 and the iMRI image was simulated from the high resolution MRI image. 


\subsection{Registration Results of SPECT and High Resolution MRI Volumes}

In Figure 1, we show an example of a successful registration of SPECT and MRI. All anatomical features including the bone marrow in the femur and pubic symphysis are well aligned in the color overlay. Using the standard algorithm in Section 2.1, the MI program successfully registered the three volume pairs. The standard parameter set was defined above with the only exception that the lower-left quadrant of the joint histogram was used for calculating MI. A successful registration was judged by the criteria defined previously.

\subsection{Image Fusion and Visualization}

In Fig. 1, we demonstrate the image fusion visualization software in a simulation of clinical usage. SPECT and high resolution MR images were acquired, transferred to a workstation, and registered prior to the "simulated" procedure. We then simulate acquiring thick iMRI slices, register them to the high resolution volume, and prepare the visualization. In this figure, one can see all. The registered images are shown in the three windows at the top line. After registration, the program creates fused images as displayed in the bottom windows.

\section{Discussion and Conclusion}

The automatic slice to volume registration algorithm is quite robust for transverse slice images covering the prostate and the registration accuracy is probably sufficiently accurate to aid iMRI-guided thermal ablation treatment. In clinical applications, physicians will always use the live-time iMRI images for needle guidance. With fused image data and visualization tools, they should be able to mentally account for any small registration errors. In addition, very often there is image evidence of cancer in MR prostate images that can perhaps be identified with the aid of functional images.

We recommend that images are obtained under comparable conditions by keeping a similar posture and by taking clinical measures to reduce rectal and bladder filling. For images acquired in quite different positions with significant deformation, we previously reported a warping registration method ${ }^{18}$ that can correct the deformations at the expense of additional complexity, time, and possibly robustness.

We think that it is feasible to include previously acquired high-resolution MRI and nuclear images into iMRI-guided treatment procedures. Image registration and fusion would provide a useful tool for the image-guided application.

Acknowledgements. The algorithm developed in this research was supported by DOD grant DAMD17-02-1-0230 to Baowei Fei, NIH grant R01-CA84433 to David L. Wilson, and ASC grant IRG91-022-06 to Zhenghong Lee. Imaging techniques were developed under the support of NIH grant R33-CA88144 to Jeffrey L. Duerk. 


\section{References}

1. J.S.Lewin, C.F.Connell, J.L.Duerk, Y.C.Chung, M.E.Clampitt, J.Spisak, G.S.Gazelle, and J.R.Haaga, "Interactive MRI-guided radiofrequency interstitial thermal ablation of abdominal tumors: Clinical trial for evaluation of safety and feasibility," Journal of Magnetic Resonance Imaging, vol. 8, pp. 40-47, 1998.

2. D.L.Wilson, A.Carrillo, L.Zheng, A.Genc, J.L.Duerk, and J.S.Lewin, "Evaluation of 3D image registration as applied to MR-guided thermal treatment of liver cancer," Journal of Magnetic Resonance Imaging, vol. 8, pp. 77-84, 1998.

3. A.Carrillo, J.L.Duerk, J.S.Lewin, and D.L.Wilson, "Semiautomatic 3-D image registration as applied to interventional MRI liver cancer treatment," IEEE Transactions on Medical Imaging, vol. 19, pp. 175-185, 2000.

4. D.B.Sodee, N.Malguria, P.Faulhaber, M.I.Resnick, J.Albert, and G.Bakale, "Multicenter ProstaScint imaging findings in 2154 patients with prostate cancer," Urology, vol. 56, pp. 988-993, 2000.

5. J.Scheidler, H.Hricak, D.B.Vigneron, K.K.Yu, D.L.Sokolov, L.R.Huang, C.J.Zaloudek, S.J.Nelson, P.R.Carroll, and J.Kurhanewicz, "Prostate cancer: Localization with threedimensional proton MR spectroscopic imaging - Clinicopathologic study," Radiology, vol. 213, pp. 473-480, 1999.

6. F.Maes, A.Collignon, D.Vandermeulen, G.Marchal, and P.Suetens, "Multimodality image registration by maximization of mutual information," IEEE Transactions on Medical Imaging, vol. 16, pp. 187-198, 1997.

7. D.B.Sodee, R.J.Ellis, M.A.Samuels, J.P.Spirnak, W.F.Poole, C.Riester, D.M.Martanovic, R.Stonecipher, and E.M.Bellon, "Prostate cancer and prostate bed SPECT imaging with ProstaScint (R): Semiquantitative correlation with prostatic biopsy results," Prostate, vol. 37, pp. 140-148, 1998.

8. A.Collignon, F.Maes, D.Delaere, D.Vandermeulen, P.Suetens, and G.Marchal, "Automated multimodality image registration using information theory,"Information Processing in Medical Imaging: Proc. 14th International Conference (IPMI'95), Computational Imaging and Vision,pp. 287-298, 1995.

9. W.H.Press, S.A.Teukolsky, W.T.Vellerling, and B.P.Flannery. Numerical Recipes in C: The Art of Scientific Computing, Second Edition. New York: The Press Syndicate of the Cambridge University, 1992.

10. B.W.Fei, J.L.Duerk, and D.L.Wilson, "Automatic 3D Registration for Interventional MRIGuided Treatment of Prostate Cancer," Computer Aided Surgery, vol. 7, pp. 257-267, 2002.

11. B.W.Fei, J.L.Duerk, D.T.Boll, J.S.Lewin, and D.L.Wilson, "Slice to volume registration and its potential application to interventional MRI guided radiofrequency thermal ablation of prostate cancer," IEEE Transactions on Medical Imaging, vol. 22, no. 4, pp. 515-525, 2003.

12. B.W.Fei, Frinkley K., and D.L.Wilson, "Comparison of Different Registration Algorithms for Interventional MRI-guided Treatment of the Prostate Cancer," Proceeding of SPIE on Medical Imaging: Visualization, Image guided procedures, and display, 2003.

13. B.W.Fei, A.Wheaton, Z.Lee, K.Nagano, J.L.Duerk, and D.L.Wilson, "Robust registration algorithm for interventional MRI guidance for thermal ablation of prostate cancer," Proceedings of SPIE Medical Imaging on Visualization, Display, and Image-Guided Procedures, vol. 4319, pp. 53-60, 2001.

14. H.M.Hudson and R.S.Larkin, "Accelerated image-reconstruction using ordered subsets of projection data," IEEE Transactions on Medical Imaging, vol. 13, pp. 601-609, 1994.

15. A.Macovski, "Noise in MRI," Magnetic Resonance in Medicine, vol. 36, pp. 494-497, 1996. 
16. R.C.Gregg and R.D.Nowak, "Noise removal methods for high resolution MRI," IEEE Nuclear Science Symposium, vol. 2, pp. 1117-1121, 1997.

17. B.W.Fei, A.Wheaton, Z.Lee, J.L.Duerk, and D.L.Wilson, "Automatic MR volume registration and its evaluation for the pelvis and prostate," Physics in Medicine and Biology, vol. 47, pp. 823-838, 2002.

18. B.W.Fei, C.Kemper, and D.L.Wilson, "A comparative study of warping and rigid body registration for the prostate and pelvic MR volumes," Computerized Medical Imaging and Graphics, vol. 27, pp. 267-281, 2003. 